\title{
MAN-MADE AREA CHANGE DETECTION BASED ON MASK WITH HJ IMAGE AND TM IMAGE
}

\author{
He xiaoying \\ National Geomatics Center of China \\ Beijing, China, 100830
}

KEYWORDS: HJ Image, TM Image, Change Detection, Man-made Area, Mask, Texture, SVM.

\begin{abstract}
:
Change detection of man-made area with HJ image and TM image is difficult mostly due to HJ image having less bands and lower spectral response. In this paper, after the image analysis of HJ image and TM image, a new change detection method based on several times mask of HJ image was used to get really changed man-made area. Several times mask was applied here to narrow the detecting object range. Firstly, coarse man-made areas of new HJ image were extracted by support vector machine (SVM) classer in the mask image based on texture and spectral extraction, while old man-made areas were picked up by SVM classer directly in TM original image. Secondly, after algebra change detection between extracted man-made areas of two images, candidate changed areas were used as mask for HJ image again, and then extractions by SVM classer in the mask images were applied to get really changed man-made area. Through these steps, a high accuracy of man-made area change detection between HJ image and TM image could be got, which had been proved by the experience and accuracy analysis.
\end{abstract}

\section{INTRODUCE}

"On demand and timely updating" of fundamental geographic databases has become one of the major tasks of Chinese national mapping agency 错误! 未找到引用源。. In the process of “on demand and timely updating", the most important thing is to get change information of geographic data quickly and efficiently. The development of remote sensing technology has promoted a good way for change detection of geographic data with its multi-scales space resolution and high time resolution. To adapt to needs of change detection nation widely, the remote sensing image data sources must meet some indexes, such as being obtained conveniently, the cost of a regular territorial coverage being affordable, enough spatial resolution, short period of covering the whole country again, etc. The environmental monitoring satellites (HJ-1A, HJ-1B), launched by our country, with $30 \mathrm{~m}$ space resolution, have rapid revisiting capability and larger field of view, which had offered images since year 2009. They are suitable for the change detention nation widely.

In the field of geographic information, many studies have been carried out around HJ CCD images and some preliminary results have been achieved 错误! 未找到引用源。, about change detection with them, there has little published research. In the previous study, change detection between images was often summarized as pixel-pixel comparison and post-classification comparison, the former being more suitable for homologous images, the latter for multi-source images 错误! 未找到引用源。

In this paper, change detection for the purpose of providing changed man-made area, including residential area and communication network, was discussed based on the latest HJ image and older TM image. In topographic map, residential area and communication network are more easily to change than other features, so they are the key points of geographic data updating. TM image has similar sensor orbit and imaging parameters with HJ image, such as viewing angles of sensors, the imaging time, imaging solar altitudes, image space resolution, etc 错误! 未找到 引用源。. Therefore, change detection between the two sensor images is feasible and of current interest. To improve accuracy of change detection, several times mask of $\mathrm{HJ}$ image was applied here to narrow the detecting object range. Firstly, coarse man-made areas from HJ image were extracted by SVM classer after mask based on texture and spectral extraction. Secondly, after algebra change detection between extracted residential areas of two images, candidate changed areas were used as masks for HJ image again, and then extractions by SVM classer in these mask images were used to get really changed man-made area. Through these steps, a high accuracy of change detection between HJ image and TM image could be obtained.

\section{ANALYSIS OF IMAGES}

Although with the same spatial resolution, the HJ CCD image has less bands and lower spectral response than the TM image. Some typical features spectral curses and statistics characteristics of two different source images after radiometric calibration were shown as follows. From left to right, the first was HJ image, the second was spectral curse of cursor location in HJ image, the third was TM image, the forth was spectral curse of cursor location in TM image.

Through the above figures and tables it can be seen, $\mathrm{HJ}$ image has a narrower radiometric value range than TM image and variances of all wavebands are smaller than those of TM image. The spectral curses of different features in HJ image have little difference with each other. Overall, the HJ image has the less radiation precision and it is difficult to distinguish different features with each other directly.

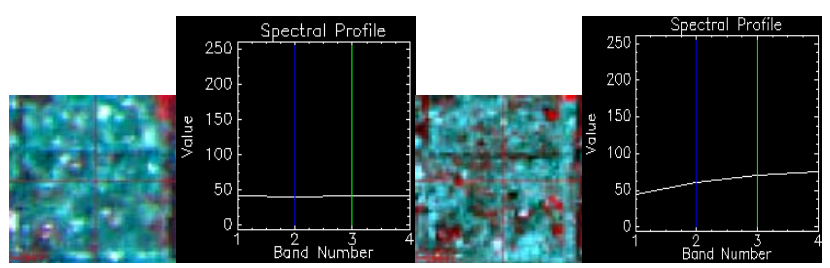

a. residential area 


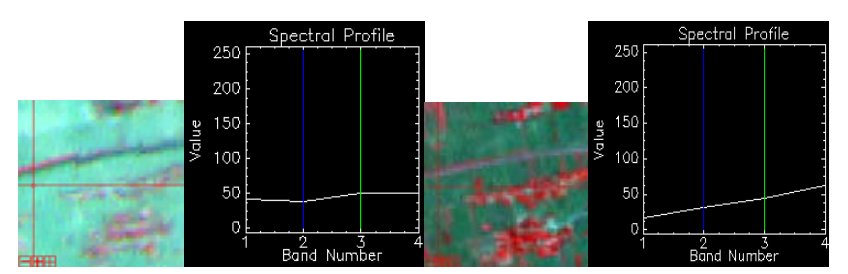

b. crop land

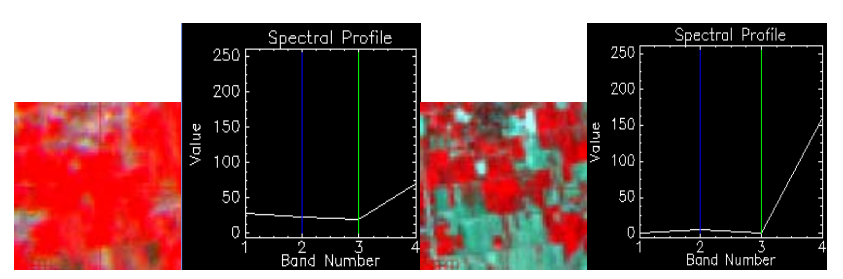

c. forest

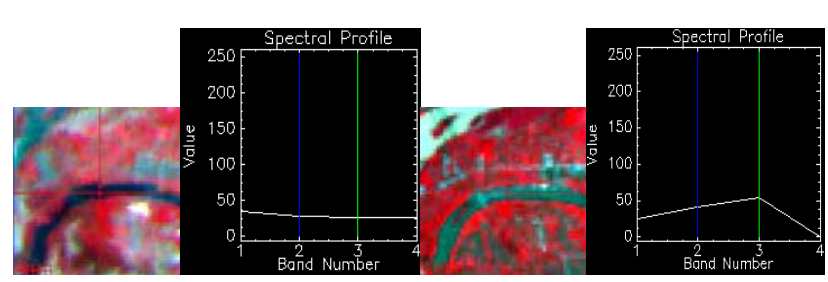

d. water

Fig.1 Spectral curses of several typical features in HJ image and TM image
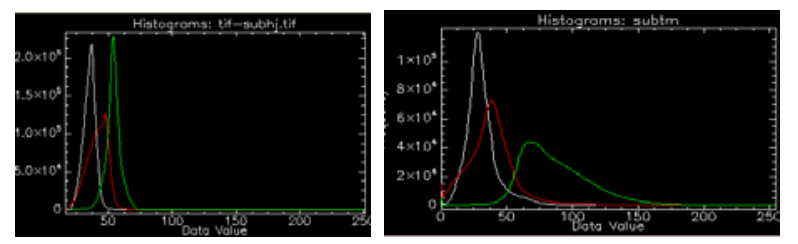

Fig.2 Histograms of 2.3.4 bands (left: from HJ image, right: from TM image)

\begin{tabular}{|c|c|c|c|c|}
\hline \multirow{2}{*}{ band } & \multicolumn{4}{|c|}{ HJ image } \\
\cline { 2 - 5 } & $\min$ & $\max$ & mean & variance \\
\hline b1 & 26 & 192 & 40.07 & 4.26 \\
\hline b2 & 18 & 198 & 35.07 & 5.03 \\
\hline b3 & 16 & 214 & 41.31 & 8.05 \\
\hline b4 & 20 & 251 & 53.63 & 5.98 \\
\hline band & \multicolumn{5}{|c|}{ TM image } \\
\cline { 2 - 5 } & min & max & mean & variance \\
\hline b1 & 0 & 226 & 15.35 & 10.07 \\
\hline b2 & 0 & 255 & 30.93 & 12.40 \\
\hline b3 & 0 & 255 & 38.10 & 18.22 \\
\hline b4 & 0 & 255 & 87.12 & 27.78 \\
\hline
\end{tabular}

Tab.1 Statistics values of HJ image and TM image

\section{CHANGE DETECTION METHOD}

From the analysis above, it is obvious that man-made area is difficult to be extracted in HJ image, especially being susceptible to confuse with crop land. So a new method applying several times mask of HJ image and several times SVM classer extraction was provided in this paper to get really changed information of man-made area between HJ image and TM image.

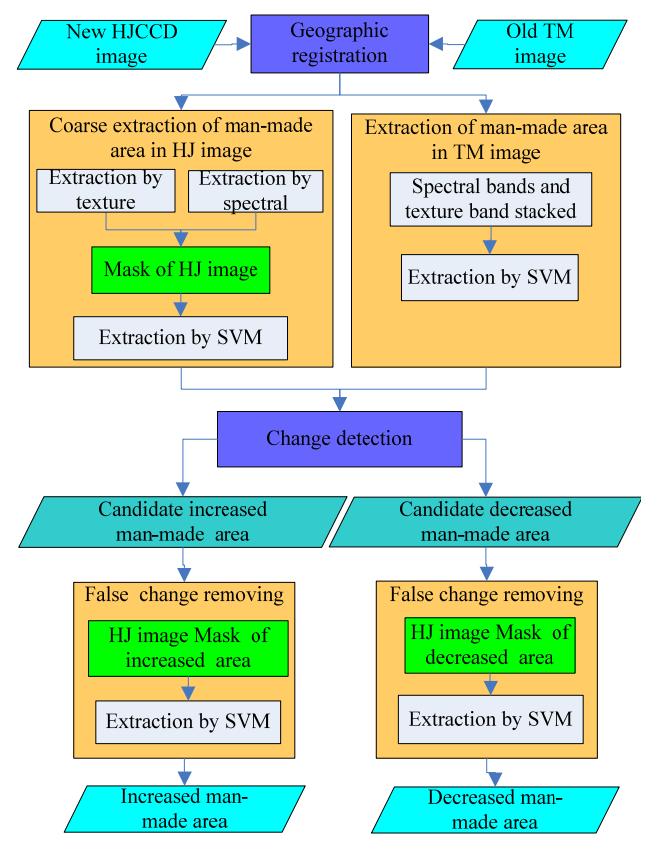

Fig.3 Flow of change detection of man-made area with HJ image and TM image

\section{CASE STUDY}

\subsection{Study area and Data}

The study was carried out in Xian City, Shanxi Province, China. The HJ image was acquired at June 29th, 2009 with path-row 7-72 for HJ1B-CCD1 and the TM image was acquired at June 13th, 2000 with path-row 127036. A subarea with $1678 * 1379$ pixels was selected for study. TM image was employed as the reference scene to which HJ image was geometric registered. After geometric rectification and, RMSE of registration between two images was about 1 pixel.

\subsection{Process of the Work}

4.2.1 Coarse Man-made Area Extraction in HJ Image: By farther analysis, man-made area was included with the buildings, roads and mixed vegetations etc. What is more, composition and construction materials of buildings and roads were different too. These characteristics lead to most of the residential area having the texture features, which had been used by many researchers 错 误! 未找到引用源。. So texture features can be use to remove other irrelevant features, such as the high reflective crop land area.

4.2.2 Extraction Based on Texture: Red band of HJ image has better performance of man-made area than others, so the red band was used to get texture image by mean of co-occurrence texture. In Fig.4, the left was original image (being shown as 
432 bands), the middle was texture image, red region was region of interesting (ROI), and the right was the texture image histogram of ROI in the middle.



a. Texture characteristic of village residential area

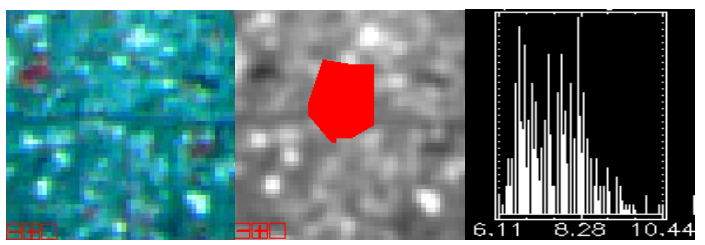

b. Texture characteristic of city residential area



c. Texture characteristic of crop land

Fig. 4 Texture characteristic of several typical features

Through experience, with threshold of 5-10.1, all village residential area and most city residential areas and roads had been extracted.

4.2.3 Extraction Based on Spectrum: Some large lump man-made area in city could not be extracted through texture characteristic. For example, the texture of follow residential area was similar to the crop land of Fig.4.

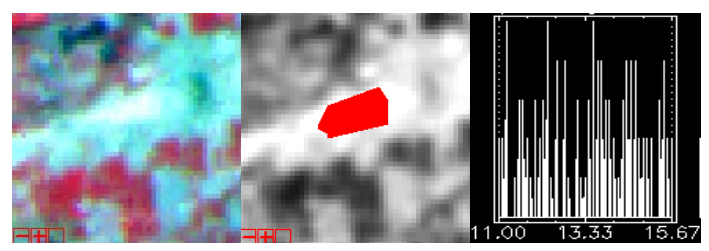

Fig.5 Texture characteristic of large lump residential area in city

Through spectrum analysis, in blue band, large lump man-made areas show high reflective property, while reflective property of crop land was lower. By this characteristic, the former could be extracted. In Fig.6, the left was original blue band image, red region was ROI, and the right was the histogram of ROI in the left.

Through experience, man-made area could be extracted with threshold range in 46-255.

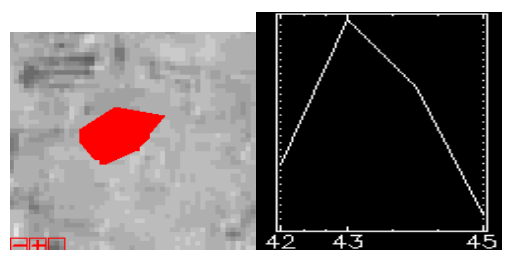

a. Corp land in blue band and its histogram

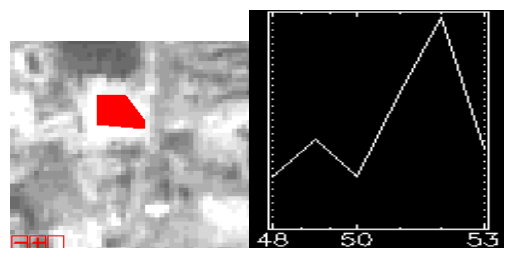

b. Man-made area in blue band and its histogram

Fig.6 Corp land and man-made area in blue band

4.2.4 Extraction by SVM Classer After Masking: After texture and spectrum extraction, all man-made area were got, at the same some other features such as shrub area was also picked up. So, the extracted result was used to mask the original HJ image and SVM classer was applied in the mask image to get coarse man-made area. The SVM classer has a good recognization effect in small samples[9,10].

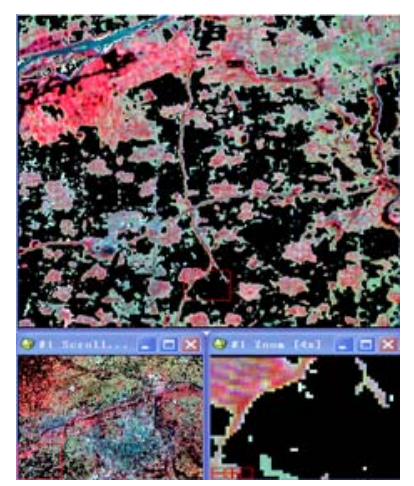

Fig.7 Man-made area mask after texture and spectrum extraction

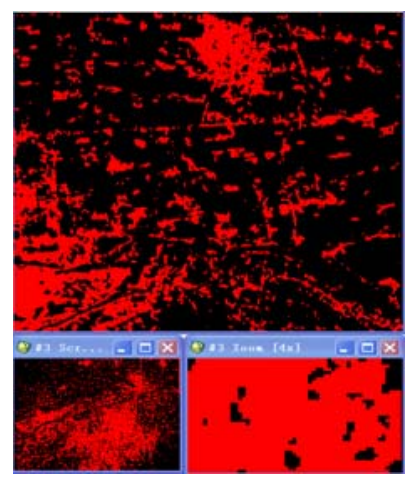

Fig.8 Coarse man-made area extracted by SVM in mask HJ image 

Man-made area in TM image was extracted by SVM classer with six original $30 \mathrm{~m}$ spectral bands and co-occurrence mean texture from red band, which had better reflect performance of man-made features.
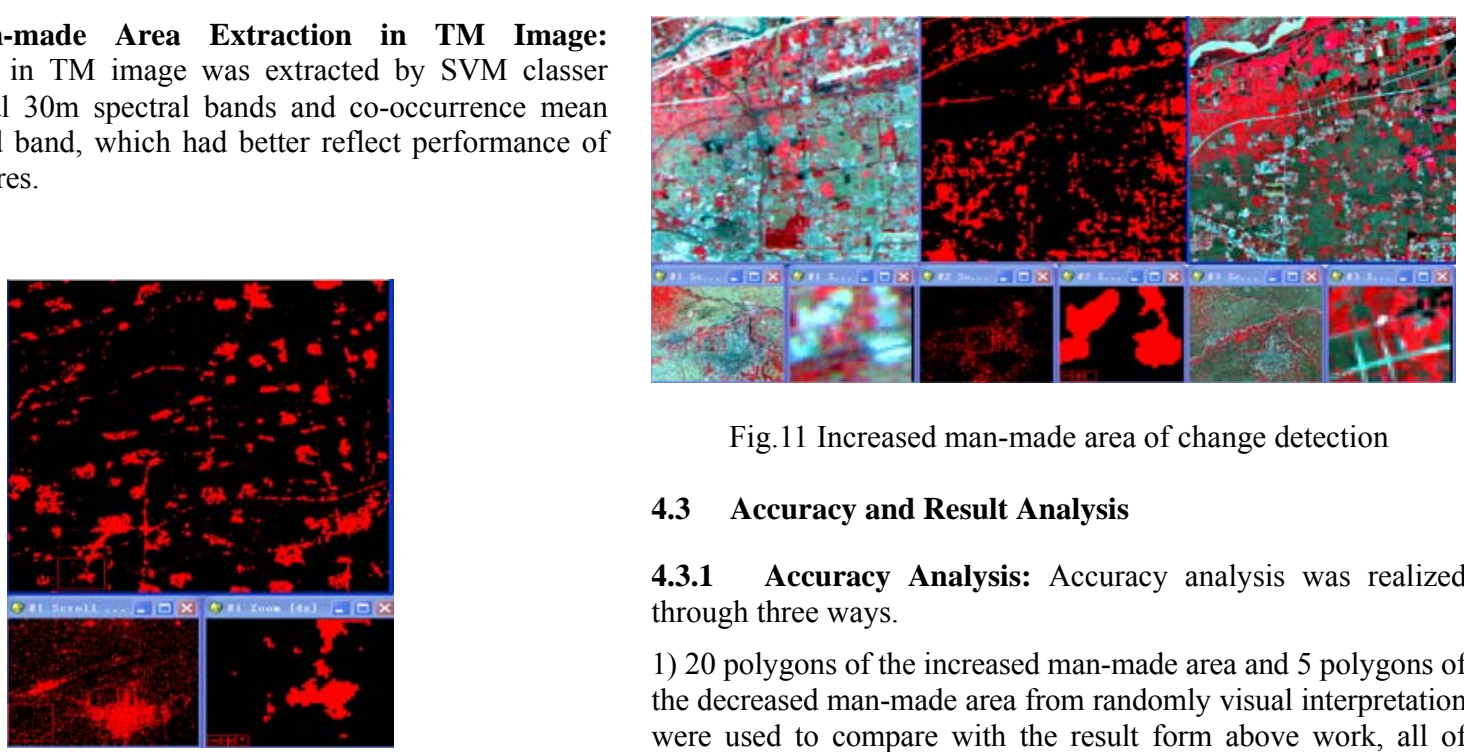

Fig.11 Increased man-made area of change detection

\subsection{Accuracy and Result Analysis}

4.3.1 Accuracy Analysis: Accuracy analysis was realized through three ways.

1) 20 polygons of the increased man-made area and 5 polygons of the decreased man-made area from randomly visual interpretation were used to compare with the result form above work, all of them were detected out in the method, and only small differences on the edge of polygons were found.

Fig.9 Man-made area extracted by SVM in TM image

4.2.6 Man-made Area Change Detection: Algebra operation was used to get increased man-made area and decreased man-made area.

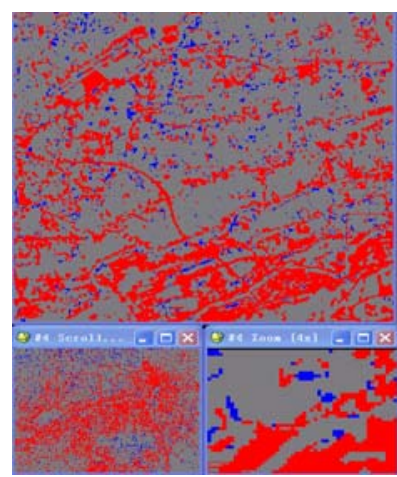

Fig.10 Change detection of man-made area (Increased man-made area in red, decreased man-made area in blue)

4.2.7 False Change Removing: Because TM image has good radiometric quantity and more bands, the man-made area extracted in old TM image was considered as the right extraction, so the false change removing was to determine whether changes of increased man-made area in the HJ image were man-made area and changes of the decreased man-made area in the HJ were not man-made area. The concrete steps were: 1)Candidate increased and decreased man-made area got form change detection were used as mask applied in original HJ images; 2)Further identified by SVM extraction in the mask image to get really changes. In the candidate increased man-made area, the extracted man-made class was considered as really increased area, while in the candidate decreased man-made area, the extracted man-made class was considered as false change detection, those non-man-made area was really decreased area.

Finally, in order to remove some too small changed polygons, majority/minority analysis and small polygons sieving were used after the SVM extraction. The result of last increased man-made area was shown as follow.
2) 30 points were laid out in changed area randomly through visual interpretation, 26 points were detection by the method, omission error $4 / 30=13.3 \%$.

3) 50 points were laid out in changed area by detection randomly $(45$ points in increased area and 5 points in decreased area), 45 points had changed really, commission error $5 / 50=10 \%$.

\subsubsection{Result Statistics:}

\begin{tabular}{|l|l|l|}
\hline & Pixel number & Area $\left(\mathrm{km}^{2}\right)$ \\
\hline Study area & 2313962 & 2082.56 \\
\hline Increased man-made area & 210811 & 189.792 \\
\hline Decreased man-made area & 18883 & 16.994 \\
\hline
\end{tabular}

Tab.2 Result of changed man-made area

Changed areas were mostly located in suburbs of Xi'an city, such as new Jingwei industrial park at the junction of Jing River and Wei River, while in village area, there was little change. The result was consistent with the pattern of city development.

\section{CONCLUSION}

From the accuracy assess, it could be drawn out that the final result of man-made area change detection was agreement with the reality change well. Because the image quality of some area in $\mathrm{HJ}$ image was poor, the direct extraction of man-made area was difficult, so mask image of texture and spectrum applied to get coarse man-made area was a practical way. After change detection, mask of changed area was applied to get a further extraction of really changed area, which was proved to get rid of false changed information really in the study.

\section{ACKNOWLEDGMENT}

The work is funded by the international scientific and technological cooperation projects 2009DFA22540. 


\section{REFERENCES}

[1] Chen Jun, Zhao Ren-liang, Wang Dong-hua. 2007, Dynamic Updating System for National Fundamental GISs: Concepts and Research Agenda. GEOMATICS WORLD, 5(5), pp. 4-9.

[2] Sun Yuan, GU Xing-fa,YU Tao, et al.. 2010, A Study of HJ-1A CCD Image Atmospheric Correction. REMOTE SENSING FOR LAND AND RESOURCES, (4):6-9.

[3] SUN Zhong-ping, XIONG Wen-cheng, WEI Bin, et al. 2010, Image Quality Eavluation of HJ-1 Stellite CCD Sensor. INFRARED (MONTHLY), 31(9), pp.30-36.

[4] Zhang Jing-cheng, Gu Xiao-he, Wang Ji-hua, et al. 2010, Analysis of consistency between HJ-CCD images and TM images in monitoring rice LAI. Transactions of the CSAE, 26(7), pp.186-193.

[5] Johnson R D ,Kasischke E S. 1998, Change vector analysis: a technique for the multi-spectral monitoring for land cover and condition. International Journal of Remote Sensing, 19(3), pp. 411-426.

[6] LI Quan LI Lin, ZHAO Xi. 2005, Urban Change Detection
Using Landsat TM Imagery. Geomatics and Information Science of Wuhan University, 30(4), pp.352-354.

[7] C. Leignel , O. Caelen, O. Debeir. 2010, DETECTING MAN-MADE STRUCTURE CHANGES TO ASSIST GEOGRAPHIC DATA PRODUCERS IN PLANNING THEIR UPDATE STRATEGY, ISPRS Archive Vol. XXXVIII, Part 4-8-2-W9, pp. 26-32.

[8] ZHANG Jian-qing, SHE Qiong,PAN Li. 2008, Change Detection of Residentialial Area by Remote Sensing Image Based on LBP/C Texture. Geomatics and Information Science of Wuhan University, 33(1), pp.7-11.

[9] Gonzalez C. R., Woods E. R.. 2002, Digital Image Processing, Second Edition. Pearson Education, Inc, pp. 425-432.

[10]M. Pal, and P. M. Mather. 2005, "Support vector machines for classification in remote sensing." International Journal of Remote Sensing, 26(5), pp. 1007-1011. 